\title{
Hypoxic regulation of glycosylation via the $N$-acetylglucosamine cycle
}

\author{
Ken Shirato, ${ }^{1}$ Kazuki Nakajima, ${ }^{1}$ Hiroaki Korekane, ${ }^{1}$ Shinji Takamatsu, ${ }^{1}$ Congxiao Gao, ${ }^{1}$ Takashi Angata, ${ }^{2}$ \\ Kazuaki Ohtsubo ${ }^{1}$ and Naoyuki Taniguchi ${ }^{1,3, *}$ \\ 'Department of Disease Glycomics, Institute of Scientific and Industrial Research, Osaka University, 8-1 Mihogaoka, Ibaraki, Osaka 567-0047, Japan \\ 2Department of Biochemistry and Molecular Biology, Graduate School of Medicine, Osaka University, 2-2 Yamadaoka, Suita, Osaka 565-0871, Japan \\ ${ }^{3}$ Systems Glycobiology Research Group, Advanced Science Institute, RIKEN, 2-1 Hirosawa, Wako, Saitama 351-0198, Japan
}

(Received 10 November, 2010; Accepted 12 November, 2010)

\begin{abstract}
Glucose is an energy substrate, as well as the primary source of nucleotide sugars, which are utilized as donor substrates in protein glycosylation. Appropriate glycosylation is necessary to maintain the stability of protein, and is also important in the localization and trafficking of proteins. The dysregulation of glycosylation results in the development of a variety of disorders, such as cancer, diabetes mellitus and emphysema. Glycosylation is kinetically regulated by dynamically changing the portfolio of glycosyltransferases, nucleotide sugars, and nucleotide sugar transporters, which together form a part of what is currently referred to as the "Glycan cycle". An excess or a deficiency in the expression of glycosyltransferases has been shown to alter the glycosylation pattern, which subsequently leads to the onset, progression and exacerbation of a number of diseases. Furthermore, alterations in intracellular nucleotide sugar levels can also modulate glycosylation patterns. It is observed that pathological hypoxic microenvironments frequently occur in solid cancers and inflammatory foci. Hypoxic conditions dramatically change gene expression profiles, by activating hypoxia-inducible factor-1, which mediates adaptive cellular responses. Hypoxia-induced glycosyltransferases and nucleotide sugar transporters have been shown to modulate glycosylation patterns that are part of the mechanism associated with cancer metastasis. Hypoxia-inducible factor-1 also induces the expression of glucose transporters and various types of glycolytic enzymes, leading to shifts in glucose metabolic patterns. This fact strongly suggests that hypoxic conditions are an important factor in modulating various nucleotide sugar biosynthetic pathways. This review discusses some of the current thinking of how hypoxia alters glucose metabolic fluxes that can modulate cellular glycosylation patterns and consequently modify cellular functions, particularly from the standpoint of the $\mathrm{N}$-acetylglucosamine cycle, a part of the "Glycan cycle".
\end{abstract}

Key Words: glycan cycle, hypoxia, sugar metabolism, nucleotide sugar, $\mathrm{N}$-acetylglucosaminyltransferase

Biosynthetic Pathways of Uridine Diphosphate (UDP)-Nacetylglucosamine (GICNAC)

Clucose is used as an energy source to produce adenosine $\checkmark$ triphosphate (ATP) through metabolic processes, such as glycolysis and cellular respiration. The free energy, generated by the hydrolytic cleavage of the high-energy phosphate linkages of ATP, is then used to drive various cellular activities. However, glucose is essential not only as an energy source but also a metabolic precursor of nucleotide sugars, which are utilized as donor substrates in protein glycosylation. Glycosylation, one of the most abundant posttranslational modification reactions, is necessary, in terms of protein stability, as well as the localization and trafficking of the proteins. Because approximately $50 \%$ of all known proteins in eukaryotes are glycosylated, ${ }^{(1)}$ the fine tuning of glycosylation is important for maintaining biological systems in a normal context.

Glucose, after its incorporation into the cells via glucose transporters (GLUTs), is initially converted to glucose-6-phosphate by hexokinases, and a portion of the glucose-6-phosphate is then metabolized via the pentose phosphate pathway, and this leads to production of nucleotides, including uridine triphosphate (UTP), guanosine triphosphate (GTP) and cytidine triphosphate (CTP), which are structural components of diverse nucleotide sugars, such as UDP-GlcNAc, UDP- $N$-acetylgalactosamine and UDPglucuronic acid, guanosine diphosphate (GDP)-mannose and GDPfucose, and cytidine monophosphate (CMP)- $N$-acetylneuraminic acid (CMP-NeuAc), respectively, or is used in glycogen synthesis to store surplus glucose in the form of glycogen in cells (Fig. 1).

In addition, under steady state conditions, approximately $1-3 \%$ of the glucose-6-phosphate enters the hexosamine biosynthetic pathway after conversion to fructose-6-phosphate, ${ }^{(2)}$ while the remaining glucose-6-phosphate is metabolized by glycolysis after conversion to fructose-6-phosphate (Fig. 1). In the hexosamine biosynthetic pathway, fructose-6-phosphate is first converted into glucosamine-6-phosphate by glutamine-fructose-6-phosphate amidotransferases (GFAT), the rate-limiting enzyme of the hexosamine biosynthetic pathway; and the glucosamine-6phosphate is then acetylated by glucosamine-6-phosphate $N$ acetyltransferases (GNA) to form GlcNAc-6-phosphate, which is isomerized to GlcNAc-1-phosphate and uridinylated by the action of UDP-GlcNAc pyrophosphorylases (UAP) (Fig. 1). The synthesized UDP-GlcNAc is transported to the Golgi apparatus via the UDP-GlcNAc transporters, ${ }^{(3)}$ and is then utilized as a donor substrate for the $\mathrm{N}$ - and $O$-linked glycosylation of extracellular and membrane proteins; alternatively, it is utilized in the cytosol for the $O$-GlcNAc modification ( $O$-GlcNAcylation) of intracellular proteins.

\section{Biological Activities of $\mathbf{N}$-Acetylglucosaminyltransferases (GnTs)}

In mammalian cells, proteins are modified with a variety of glycans, which can be classified into two major groups, $N$ - and $O$ glycans. ${ }^{(1)} N$-glycans are linked to certain asparagine residues of proteins that contain the Asn-X-Ser/Thr motif, whereas $O$-glycans are attached to a subset of serine and threonine residues. ${ }^{(4)}$ The addition of GlcNAc from UDP-GlcNAc to $N$ - and $O$-glycans and $O$-GlcNAcylation is catalyzed by enzymatic activity of GnTs and $O$-linked $N$-acetylglucosaminyltransferase (OGT), localized

*To whom correspondence should be addressed. E-mail: tani52@wd5.so-net.ne.jp 


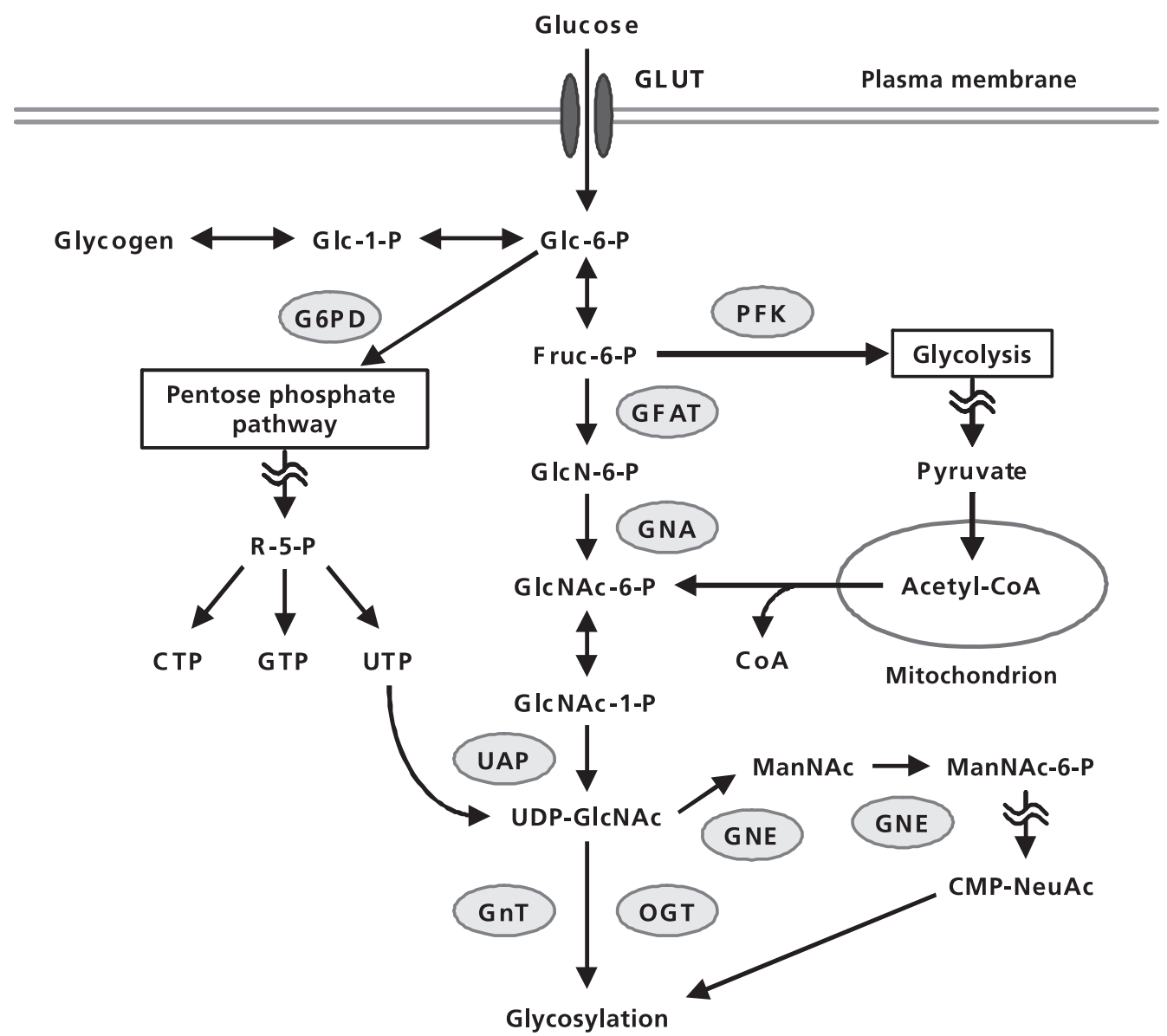

Fig. 1. Outline of UDP-GICNAC synthetic pathways. UDP-GICNAC is synthesized by three glucose metabolic pathways, such as the pentose phosphate pathway and subsequent nucleotide synthesis, hexosamine biosynthetic pathway, and glycolysis, supplying UTP, glcosamine and acetyl group, respectively. GLUT, Glucose transporter; Glc, glucose; P, phosphate; R, ribose; CTP, cytidine triphosphate; GTP, guanosine triphosphate; UTP, uridine triphosphate; GnT, N-acetylglucosaminyltransferase; OGT, O-linked GnT; Fruc, fructose; GlcN, glucosamine; GlcNAc, $N$-acetylglucosamine; CoA, coenzyme A; ManNAc, $N$-acetylmannose; NeuAc, $N$-acetylneuraminic acid; G6PD, glucose-6-phosphate dyhydrogenase; GFAT, glutamine-Fruc-6-P amidotransferase; GNA, Glc-6-P N-acetyltransferase; UAP, UDP-GICNAc pyrophosphorylase; PFK, phosphofructokinase; GNE, UDP-GIcNAc 2-epimerase/ ManNAc kinase.

in the Golgi apparatus and cytosol, respectively. The formation of branched structures and subsequent elongation and processing of $\mathrm{N}$-glycans by various types of GnTs alter the biological functions of the glycoproteins ${ }^{(5)}$ and are associated with a variety of disorders, such as cancer and diabetes mellitus. ${ }^{(6,7)}$

As shown in Fig. 2, with respect to the GlcNAc cycle, the Golgi apparatus contains at least two enzymes, GnT-V and GnT-III, which play key roles in the processing of $N$-glycans.

GnT-V catalyzes the transfer of a GlcNAc unit from UDPGlcNAc to a $\alpha 1,6$-mannose in $N$-glycans to form a $\beta 1,6$-branched $N$-glycans, thus elongating the polylactosamine structure (Fig. 2), which controls cell surface receptor residency and is associated with some of the invasive characteristics associated with cancer cells. ${ }^{(1,8,9)}$ Indeed, the overexpression of GnT-V has been reported to promote integrin $\alpha_{5} \beta_{1}$-mediated cell migration. ${ }^{(10)}$ It has been also reported that GnT-V activity and the levels of $\beta 1,6$-branched $N$-glycans were increased in highly metastatic cancer cell lines. ${ }^{(11,12)}$

On the contrary, GnT-III catalyzes the addition of a GlcNAc unit in a $\beta 1,4$-linkage to the mannose residue at the base of the trimannosyl core of an $N$-glycan, to produce a so-called "bisecting GlcNAc" (Fig. 2). ${ }^{(13,14)}$ The introduction of a bisecting GlcNAc prevents the formation of a branched structure in $N$-glycans, since other GnTs are not able to utilize an $N$-glycan with a bisecting GlcNAc as a substrate. ${ }^{(15)}$ The overexpression of GnT-III
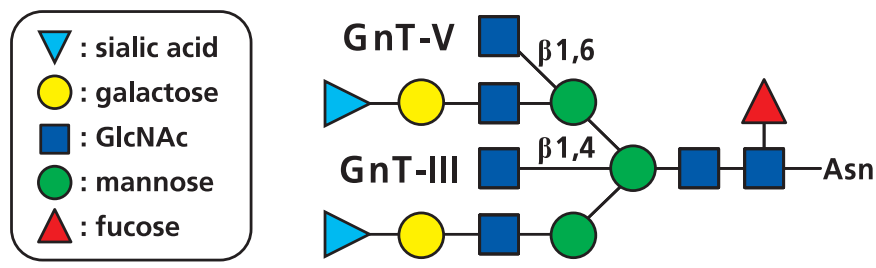

Fig. 2. Glycosylation reactions catalyzed by the actions by GnT-III and GnT-V. The enzymatic products by GnT-III and GnT-V are designated as bisecting GIcNAc and $\beta 1,6$ GlcNAc branch, respectively.

suppresses $\alpha_{5} \beta_{1}$ integrin-mediated cell spreading, migration and the phosphorylation of focal adhesion kinase (FAK). ${ }^{(16)}$ These results suggest that GnT-III has antagonizing effects against GnT-V.

Additionally, Ohtsubo et al. ${ }^{(17)}$ demonstrated that a loss of GnTIVa, which catalyzes the formation of a $\beta 4$ GlcNAc linkage on the $\alpha 3$-linked mannose of $N$-glycans, attenuates the half-life of GLUT2 on the pancreatic $\beta$-cell surface, and the resulting impairment of glucose-stimulated insulin secretion leads to metabolic dysfunctions that are characteristic of type 2 diabetes. 


\section{Regulation of Glycosylation via GIcNAc Cycle}

Furthermore, alterations in intracellular nucleotide sugar levels can also modulate glycosylation patterns (Fig. 3), ${ }^{(18)}$ because GnTs have different $\mathrm{Km}$ values for UDP-GlcNAc. Elevated intracellular UDP-GlcNAc enhances the activities of GnT-V and GnT-IV, which have higher Km values; however, low levels diminished the activities. In fact, it was demonstrated that the formation of $\beta 1,6$-branched $N$-glycans in Jurkat T-cells is stimulated by supplementation of metabolic precursors of the hexosamine biosynthetic pathway, including glucose, GlcNAc, glutamine, acetoacetate, ammonia and uridine, and regulates autoimmune reactions of T-cells. ${ }^{(19)}$ Furthermore, hyperglycemia involves an enhancement in the hexosamine biosynthetic pathway activity and $O$ GlcNAcylation levels, resulting in an enhanced susceptibility to apoptosis of pancreatic $\beta$-cells. ${ }^{(20)}$ Thus, glycosylation is affected not only by the levels of expression of a single GnT enzyme but rather by a magnitude factors, including the levels of monooligosaccharides, nucleotide sugars, and nucleotide sugar transporters, the localization of GnTs and nucleotide sugar levels in the Golgi, transcription factors, the pro-form and mature form of glycoproteins, and the structure of cell surface glycoproteins (Fig. 3). ${ }^{(8)}$ These components of the "GlcNAc cycle", a part of the "Glycan cycle", should be considered in detecting changes in dynamic glycan metabolism (Fig. 3). ${ }^{(8)}$

\section{Hypoxia and Glycosylation}

Pathological hypoxic microenvironments, which are observed in many cases of solid cancers and inflammatory foci, dramatically change gene expression profiles, and confer various malignant characteristics to cancer cells. Hypoxia-inducible factor-1
(HIF-1) plays key roles in adaptive responses of cells under hypoxic conditions through its transcription activity. The HIF-1 protein is a heterodimer, composed of a constitutively expressed subunit of HIF-1 $\beta$ and an oxygen-dependently regulated subunit of HIF- $1 \alpha^{(21)}$ (Fig. 4). The expression of HIF-1 $\alpha$ is predominantly regulated at posttranslational level. Under oxygen-sufficient conditions, prolyl residues 402 and 564 in the N-terminal domain of HIF- $1 \alpha$ protein are hydroxylated by the actions of prolyl hydroxylases (PHD) in an oxygen-dependent manner (Fig. 4). This enables the binding of E3 ubiquitin ligase Von Hippel-Lindau to HIF-1 $\alpha$, and leads to proteasomal degradation of the HIF-1 $\alpha$ protein (Fig. 4). On the contrary, under hypoxic conditions, because enzymatic activities of PHDs are reduced, the HIF-1 $\alpha$ protein is stabilized and is translocated to the nucleus, where induces gene expression (Fig. 4). Furthermore, in this process, it has been demonstrated that reactive oxygen species (ROS) generated from mitochondria under conditions of hypoxia is required for stabilization of the HIF-1 $\alpha$ protein. ${ }^{(22)}$

It is well known that hypoxic microenvironments dramatically shift the pattern of intracellular glucose metabolism from aerobic cellular respiration to anaerobic glycolysis. Under hypoxic conditions, the expression of GLUT1 and various types of the ratelimiting enzymes of glycolysis, such as phosphofructokinases (PFK), aldolases (ALD), phosphoglycerate kinases (PGK), enolases (ENO) and lactate dehydrogenases (LDH), are markedly transactivated by HIF-1 (Fig. 4). The resulting increased glucose uptake and activation of glycolysis are highly important compensatory responses against a reduction in intracellular ATP levels due to the hypoxic inhibition of cellular respiration.

In a previous study, Koike et al. ${ }^{(23)}$ reported that hypoxia increases the levels of cell surface sialyl Lewis $\mathrm{x}$ and sialyl Lewis a determinants, which are specific ligands for E-selectin, and

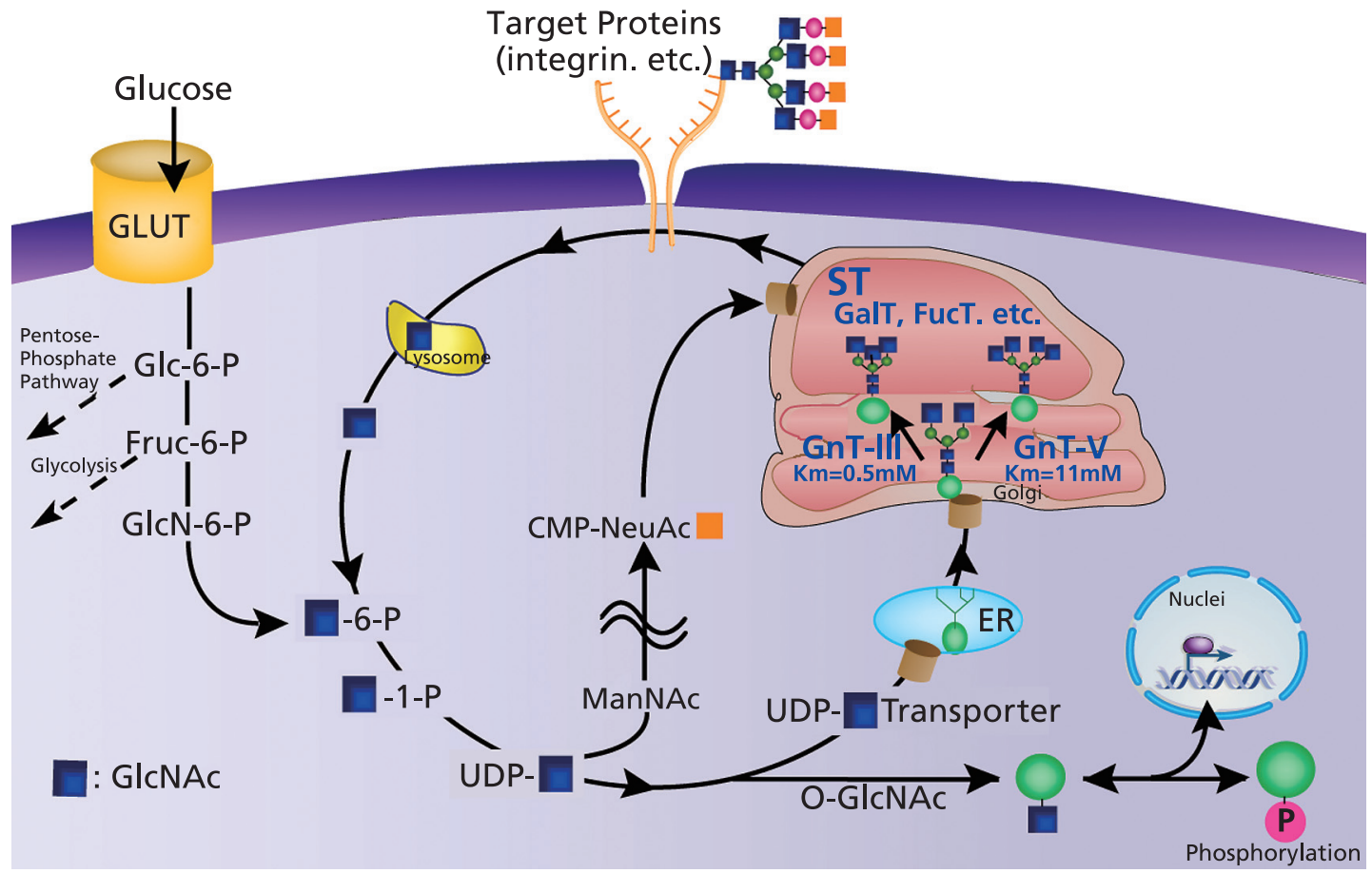

Fig. 3. Schmatic drawing of GICNAc cycle (modified version of Ref. 18). Glycosylation is affected by various factors as described in text, and the proform is converted to the mature form of glycoproteins by various glycosyltransferases and then localized on the cell surface such as integrin. The intracellular levels of UDP-GIcNAC and its precursor metabolites in sugar metabolism affect branching formation of $N$-linked glycans, because GICNAC transferases have different apparent $\mathrm{Km}$ values for UDP-GIcNAC. Also, the addition of $\mathrm{O}-\mathrm{GlcNAC}$ to proteins is highly responsive to UDPGIcNAc levels, and $O$-GlcNAclyated or phosphoylated proteins function in a reciprocal relationship as a transcriptional regulator. GnT, $N$ acetylglucosaminyltransferase; ST, sialyltransferase; GalT, galactosaminyltransferase; FucT, fucosyltransferase; GLUT, glucose transporter; GIc-6-P, glucose-6-phosphate; Fruc-6-P, fructose-6-phosphate; GICN-6-P, glucosamine-6-phosphate; ER, endoplasmic reticulum. 


\section{normoxia}

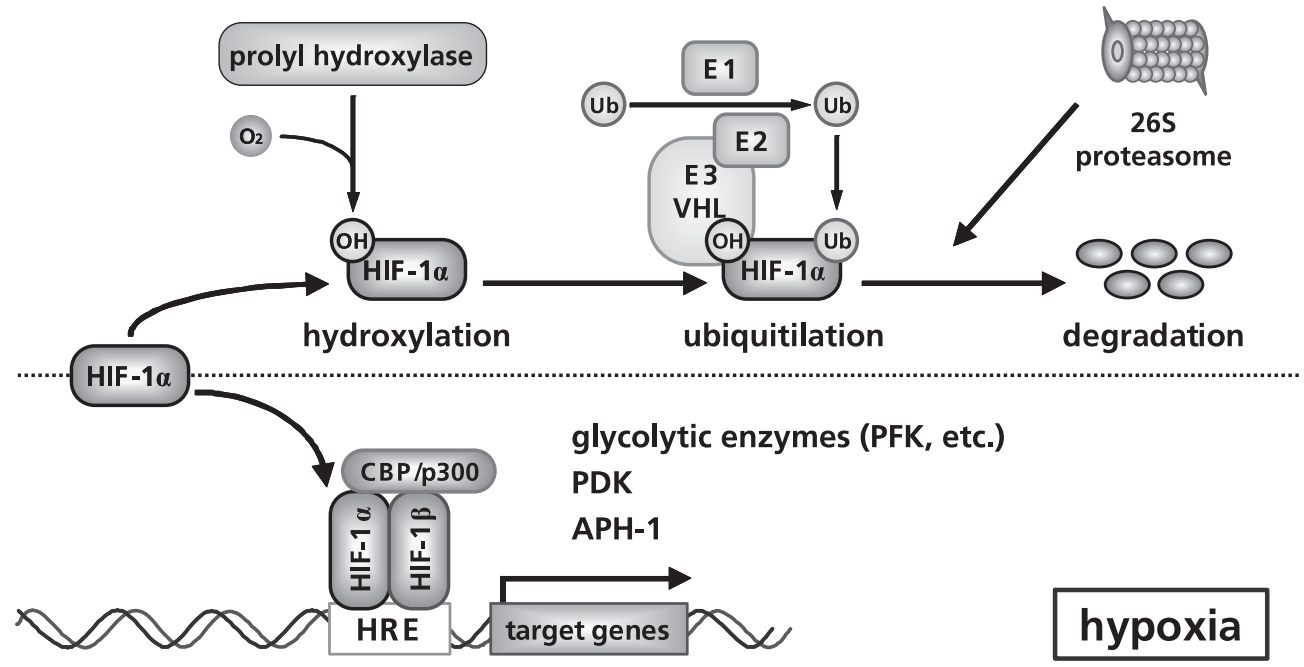

Fig. 4. Typical expression regulatory mechanism of HIF-1 protein and its possible roles in hypoxia-induced reduction in GICNAc cycle activity. HIF-1 activates a variety of proteins responsible for adaptation to hypoxia, including glycolytic enzymes (phosphofructokinase (PFK) etc.), through its transcription activity. HIF-1 also activates the transcription of pyruvate dehydrogenase kinases (PDK), which neutralize the activity of pyruvate dehydrogenase. (26) This may lead to the suppression of acetyl-CoA production and result in reduction in intracellular UDP-GIcNAc level. The anterior pharynx-defective (APH)-1, a component of $\gamma$-secretase induced by HIF-1, increases the activity of $\gamma$-secretase, ${ }^{(35)}$ which has been shown to cleave GnT-V protein residing at Golgi lumen and induce extracellular secretion of GnT-V. ${ }^{(33,34)}$

also increases the transcription of fucosyltransferase VII (FUT7), sialyltransferase ST3Gal-I and UDP-Gal transporter 1, which are involved in the synthesis of the carbohydrate ligands of endothelial E-selectin. In contrast, hypoxia dramatically shifts the glucose metabolic pattern, which should affect intracellular nucleotide sugar levels, particularly UDP-GlcNAc, and would lead to alterations in glycan structures and cellular functions. Therefore, how hypoxia alters glucose metabolic fluxes that can modulate cellular glycosylation patterns and consequently modify cellular functions, are discussed, with emphasis on the contribution of the GlcNAc cycle.

\section{Hypoxia and UDP-GICNAC}

It is unclear how hypoxic microenvironments affect the biosynthetic machinery of the nucleotide sugars. At present, there is only one report dealing with a regulatory mechanism of the hexosamine biosynthetic pathway in response to hypoxia. Manzari et al. ${ }^{(24)}$ demonstrated that hypoxia increased both mRNA and the expressions of the corresponding protein, GFAT, the rate-limiting enzyme of the hexosamine biosynthetic pathway. This study concluded that the transcriptional activity of the GFAT gene is directly regulated by HIF-1. We have also confirmed, using RAW264.7 cells (a murine macrophage cell line), that hypoxic exposure results in an increase in the mRNA levels of GFAT1 but the mRNA levels of other enzymes of the hexosamine biosynthetic pathway downstream of GFAT1, such as GNA1, UAP1 and GlcNAc kinase (NAGK) remain unchanged (unpublished data). These results imply that the biosynthesis of UDPGlcNAc should be promoted in cells under hypoxic conditions. Thus, we recently determined changes in intracellular UDPGlcNAc levels in response to hypoxia, using ion-pair reversedphase high performance liquid chromatography (HPLC) ${ }^{(25)}$ and liquid chromatography-mass spectrometry (LC-MS). Surprisingly, the intracellular level of UDP-GlcNAc was reduced by approximately $50 \%$ in cells exposed to hypoxia (unpublished data). These findings suggest that activation of the hexosamine biosynthetic pathway in response to hypoxia is not reflected in the intracellular abundance of UDP-GlcNAc.

In order to clarify hypoxia-induced alterations in the synthetic pathway of UDP-GlcNAc, we analyzed changes in the metabolic flow of glucose in response to hypoxia using unpublished LC-MS methods after labeling the starting glucose at C-6 with ${ }^{13} \mathrm{C}$. The ratio of metabolic flow was calculated from the intensity of the isotopic pattern of ${ }^{13} \mathrm{C}_{6}$-labeled nucleotide sugar in the mass spectrum. It was found that a signal intensity 6 mass units higher than that for ordinary UDP-GlcNAc in hypoxic cells was similar to that in normoxic cells. This result indicates that metabolic flow of the hexosamine biosynthetic pathway is maintained under hypoxic conditions, because the mass shift of 6 reflects UDPGlcNAc, in which the glucosamine (hexose), was replaced by a ${ }^{13} \mathrm{C}_{6}$-labeled molecule. However, the signal corresponding to 5 mass and 13 mass higher UDP-GlcNAc in hypoxic cells was markedly weaker than that in normoxic cells. The five mass shift reflects the replacement of ribose (pentose) in UDP and 13 mass shift reflects that of all of components, including ${ }^{13} \mathrm{C}_{5}$-ribose, ${ }^{13} \mathrm{C}_{6}$ glucosamine and the ${ }^{13} \mathrm{C}_{2}$-labeled acetyl group. ${ }^{13} \mathrm{C}_{5}$-ribose and ${ }^{13} \mathrm{C}_{2}$-acetyl group is incorporated by the enzymatic activity of UAP and GNA, respectively. Under oxygen-sufficient conditions, acetyl-CoA is synthesized from pyruvate, a final product of glycolysis, by pyruvate dehydrogenase. When intracellular oxygen concentration is decreased, HIF-1 activates the transcription of pyruvate dehydrogenase kinases (PDK), which neutralizes the activity of pyruvate dehydrogenase ${ }^{(26)}$ (Fig. 4). As a result, the production of acetyl-CoA and its translocation to the tricarboxylic acid (TCA) cycle is suppressed. This reaction is very important in terms of the inhibition of oxygen consumption. This also may cause the suppression in the addition of an acetyl group to glucosamine, thus leading to an overall reduction in UDP-GlcNAc production. Hisanaga et al. ${ }^{(27)}$ reported that, when neonatal rats are exposed to conditions of acute hypoxia exposure, a variety of nucleotides in the brain, such as ATP, GTP, UTP and CTP are decreased, and subsequent normoxic exposure restored these nucleotide levels. The reduction in intracellular nucleotide levels 
by hypoxic exposure may inhibit the addition of UDP to GlcNAc.

On the contrary, it is suspected that a diverging pathway of nucleotide sugar synthesis is activated in response to hypoxia. A portion of the synthesized UDP-GlcNAc has been shown to be epimerized by UDP-GlcNAc 2-epimerase (GNE), and is then supplied to the synthetic pathway of CMP-NeuAc, ${ }^{(28)}$ which leads to cell surface sialylation (Fig. 3). We found that, when RAW264.7 cells were exposed to hypoxic conditions or treated with the hypoxia-mimetic agent cobalt chloride $\left(\mathrm{CoCl}_{2}\right)$, the intracellular level of CMP-NeuAc, unlike UDP-GlcNAc, was significantly increased (unpublished data). From these results, analyses of the downstream pathway(s) of UDP-GlcNAc may reveal the mechanism behind the hypoxia-induced reduction of UDP-GlcNAc.

\section{Hypoxia and GnTs}

It is very important to clarify the mechanism by which GnTs is controlled, because the levels of such enzymes directly affect the patterns of glycosylation and is partly responsible for the GlcNAc cycle. However, to date, the transcriptional regulatory mechanism of GnT genes has not been fully elucidated. Meanwhile, our group previously demonstrated that the expression of the Mgat5 gene, encoding GnT-V, is regulated by a member of the E-twenty six (Ets) family of transcription factors, Ets-1. ${ }^{(29,30)}$ It was also reported that the expression of the ETS1 gene is regulated by HIF-1. ${ }^{(31)}$ These findings suggest the indirect induction of GnT-V expression by HIF-1 in cells that are exposed to hypoxia. However, the level of Mgat5 mRNA was relatively decreased by hypoxic exposure in the case of RAW264.7 cells (unpublished data). This may be because Ets-1 protein is constitutively expressed in monocytes and macrophages, and, as a result, the expression of Ets-1 is not inducible in response to hypoxia in these cells. ${ }^{(32)}$ These findings indicate that the expression of GnT-V is not induced in hypoxic macrophages. Furthermore, we recently reported that the enzymatic activity of GnT-V was gradually reduced along with hypoxic exposure time in RAW264.7 cells, while no GnT-III activity was detected in these cells (unpublished data). On the other hand, it has been demonstrated that the GnT-V protein, residing in the Golgi lumen, is cleaved by $\gamma$-secretase (a protease complex composed of presenilin, nicastrin, anterior pharynx-defective (APH)-1 and presenilin enhancer (PEN)-2), and the secreted type of GnT-V has angiogenesis effects. ${ }^{(33,34)}$ Wang et al. ${ }^{(35)}$ demonstrated that HIF-1 regulates the expression of APH-1A, a component of $\gamma$-secretase, and the $\gamma$-secretasemediated secretion of amyloid $\beta$ (Fig. 4). Thus, it is necessary to examine, not only transcriptional regulation but also the posttranslational modification of GnTs.

\section{Conclusion}

Hypoxia reduces the activity of a portion of the GlcNAc cycle, including intracellular UDP-GlcNAc levels and GnT-V activity. Analysis of the metabolic flow of glucose using unpublished LC-MS methods using ${ }^{13} \mathrm{C}_{6}$-labeled glucose (Nakajima et al., manuscript in preparation) indicates that the hypoxia-induced reduction in intracellular UDP-GlcNAc levels is caused by the suppression of the addition of an acetyl group to glucosamine and intracellular nucleotide levels.

To clarify physiological significances of these hypoxia-induced reductions in GlcNAc cycle activity, it is essential to analyze whether or not the levels of $\beta 1,6$-branced $N$-glycans of the target cell surface proteins are reduced. Furthermore, a better understanding of the mechanisms associated with GlcNAc cycle inactivation in response to hypoxia is important, because recovery experiments of GlcNAc cycle activities are essential in terms of identifying whether the resultant carbohydrate structural alterations contribute the hypoxia-induced changes in cellular function. Thus, it needs glucose metabolomic analysis regarding the UDPGlcNAc biosynthetic pathways, including the pentose phosphate pathway and subsequent nucleotide synthesis, hexosamine biosynthetic pathway, and glycolysis. Finally, other components of the glycan cycle, including nucleotide sugar transporters, the localization of GnTs and nucleotide sugar levels in the Golgi should also be considered, and an understanding of the glycan cycles will promise further advancements in the future for the field of glycobiology. ${ }^{(8)}$

\section{Acknowledgments}

This work was supported by Grant-in-Aid for Scientific Research (A), 20249018, from the Ministry of Education, Culture, Sports, Science and Technology, Japan.

\section{Abbreviations}

$\begin{array}{ll}\text { ALD } & \text { aldolases } \\ \text { APH } & \text { anterior pharynx-defective } \\ \text { ATP } & \text { adenosine triphosphate } \\ \text { CMP } & \text { cytidine monophosphate }\end{array}$

CMP-NeuAc cytidine monophosphate- $N$-acetylneuraminic acid

CTP cytidine triphosphate

ENO enolases

Ets E-twenty six

FAK focal adhesion kinase

FUT7 fucosyltransferase VII

GDP guanosine diphosphate

GFAT glutamine-fructose-6-phosphate amidotransferases

GlcNAc $\quad N$-acetylglucosamine

GLUTs glucose transporters

GNA glucosamine-6-phosphate $N$-acetyltransferases

GNE UDP-GlcNAc 2-epimerase

GnT $N$-Acetylglucosaminyltransferases

GTP guanosine triphosphate

HIF-1 hypoxia-inducible factor-1

HPLC reversed-phase high performance liquid chromatography

LC-MS liquid chromatography-mass spectrometry

LDH lactate dehydrogenases

NAGK GlcNAc kinase

O-GlcNAcylation O-GlcNAc modification

OGT O-linked $N$-acetylglucosaminyltransferase

PDK pyruvate dehydrogenase kinases

PEN-2 presenilin enhancer-2

PFK phosphofructokinases

PGK phosphoglycerate kinases

PHD prolyl hydroxylases

ROS reactive oxygen species

TCA tricarboxylic acid

UAP UDP-GlcNAc pyrophosphorylases

UDP Uridine Diphosphate

UDP-GlcNAc UDP- $N$-acetylglucosamine

UTP uridine triphosphate

\section{References}

$1 \mathrm{Gu}$ J, Taniguchi N. Potential of N-glycan in cell adhesion and migration as either a positive or negative regulator. Cell Adh Migr 2008; 2: 243-245.

2 Cheng DW, Jiang Y, Shalev A, Kowluru R, Crook ED, Singh LP. An analysis of high glucose and glucosamine-induced gene expression and oxidative stress in renal mesangial cells. Arch Physiol Biochem 2006; 112: 189-218.

3 Ishida N, Kawakita M. Molecular physiology and pathology of the nucleotide 
sugar transporter family (SLC35). Pflügers Arch 2004; 447: 768-775.

4 Schachter $\mathrm{H}$. The joys of HexNAc. The synthesis and function of $\mathrm{N}$ - and $\mathrm{O}$ glycan branches. Glycoconj J 2000; 17: 465-483.

5 Stanley P, Schachter H, Taniguchi N. N-glycans. In: Varki A, Cummings RD, Esko JD, and et al, eds. Essentials of Glycobiology, 2nd edn., New York: Cold Spring Harbor Laboratory Press, 2009; 101-114.

6 Taniguchi N, Miyoshi E, Gu J, Honke K, Matsumoto A. Decoding sugar functions by identifying target glycoproteins. Curr Opin Struct Biol 2006; 16: 561-566.

7 Taniguchi N, Gu J, Takahashi M, Miyoshi E. Functional glycomics and evidence for gain- and loss-of-functions of target proteins for glycosyltransferases involved in $\mathrm{N}$-glycan biosynthesis: their pivotal roles in growth and development, cancer metastasis and antibody therapy against cancer. Proc Japan Acad Ser B 2004; 80: 82-91.

8 Taniguchi N. From the $\gamma$-glutamyl cycle to the glycan cycle: a road with many turns and pleasant surprises. J Biol Chem 2009; 284: 34469-34478.

9 Partridge EA, Le Roy C, Di Guglielmo GM, and et al. Regulation of cytokine receptors by Golgi $N$-glycan processing and endocytosis. Science 2004; 306: $120-124$.

10 Guo HB, Lee I, Kamar M, Akiyama SK, Pierce M. Aberrant $N$-glycosylation of $\beta_{1}$ integrin causes reduced $\alpha_{5} \beta_{1}$ integrin clustering and stimulates cell migration. Cancer Res 2002; 62: 6837-6845.

11 Asada M, Furukawa K, Segawa K, Endo T, Kobata A. Increased expression of highly branched $N$-glycans at cell surface is correlated with the malignant phenotypes of mouse tumor cells. Cancer Res 1997; 57: 1073-1080.

12 Pocheć E, Lityńska A, Amoresano A, Casbarra A. Glycosylation profile of integrin $\alpha_{3} \beta_{1}$ changes with melanoma progression. Biochim Biophys Acta 2003; 1643: 113-123.

13 Nishikawa A, Ihara Y, Hatakeyama M, Kangawa K, Taniguchi N. Purification, cDNA cloning, and expression of UDP- $N$-acetylglucosamine: $\beta$-D-mannoside $\beta-1,4 N$-acetylglucosaminyltransferase III from rat kidney. J Biol Chem 1992; 267: 18199-18204.

14 Ihara Y, Nishikawa A, Tohma T, Soejima H, Niikawa N, Taniguchi N. cDNA cloning, expression, and chromosomal localization of human $N$-acetylglucosaminyltransferase III (GnT-III). J Biochem 1993; 113: 692-698.

15 Schachter H. Biosynthetic controls that determine the branching and microheterogeneity of protein-bound oligosaccharides. Adv Exp Med Biol 1986; 205: 53-85.

16 Isaji T, Gu J, Nishiuchi R, and et al. Introduction of bisecting GlcNAc into integrin $\alpha_{5} \beta_{1}$ reduces ligand binding and down-regulates cell adhesion and cell migration. J Biol Chem 2004; 279: 19747-19754.

17 Ohtsubo K, Takamatsu S, Minowa MT, Yoshida A, Takeuchi M, Marth JD. Dietary and genetic control of glucose transporter 2 glycosylation promotes insulin secretion in suppressing diabetes. Cell 2005; 123: 1307-1321.

18 Taniguchi N. A sugar-coated switch for cellular growth and arrest. Nat Chem Biol 2007; 3: 307-309.

19 Grigorian A, Lee SU, Tian W, and et al. Control of T Cell-mediated autoimmunity by metabolite flux to $\mathrm{N}$-glycan biosynthesis. J Biol Chem 2007; 282: 20027-20035.

20 D'Alessandris C, Andreozzi F, Federici M, and et al. Increased $O$-glycosylation of insulin signaling proteins results in their impaired activation and enhanced susceptibility to apoptosis in pancreatic $\beta$-cells. FASEB J 2004; 18: 959-961.

21 Semenza GL. Regulation of oxygen homeostasis by hypoxia-inducible factor 1. Physiology (Bethesda) 2009; 24: 97-106.

22 Emerling BM, Platanias LC, Black E, Nebreda AR, Davis RJ, Chandel NS. Mitochondrial reactive oxygen species activation of p38 mitogen-activated protein kinase is required for hypoxia signaling. Mol Cell Biol 2005; 25: 4853-4862.

23 Koike T, Kimura N, Miyazaki K, and et al. Hypoxia induces adhesion molecules on cancer cells: A missing link between Warburg effect and induction of selectin-ligand carbohydrates. Proc Natl Acad Sci USA 2004; 101: 81328137.

24 Manzari B, Kudlow JE, Fardin P, and et al. Induction of macrophage glutamine: fructose-6-phosphate amidotransferase expression by hypoxia and by picolinic acid. Int J Immunopathol Pharmacol 2007; 20: 47-58.

25 Nakajima K, Kitazume S, Angata T, and et al. Simultaneous determination of nucleotide sugars with ion-pair reversed-phase HPLC. Glycobiology 2010; 20: $865-871$.

26 Kim JW, Tchernyshyov I, Semenza GL, Dang CV. HIF-1-mediated expression of pyruvate dehydrogenase kinase: a metabolic switch required for cellular adaptation to hypoxia. Cell Metab 2006; 3: 177-185.

27 Hisanaga K, Onodera H, Kogure K. Changes in levels of purine and pyrimidine nucleotides during acute hypoxia and recovery in neonatal rat brain. $J$ Neurochem 1986; 47: 1344-1350.

28 Keppler OT, Hinderlich S, Langner J, Schwartz-Albiez R, Reutter W, Pawlita M. UDP-GlcNAc 2-epimerase: a regulator of cell surface sialylation. Science 1999; 284: 1372-1376.

29 Kang R, Saito H, Ihara Y, and et al. Transcriptional regulation of the $\mathrm{N}$ acetylglucosaminyltransferase $\mathrm{V}$ gene in human bile duct carcinoma cells (HuCC-T1) is mediated by Ets-1. J Biol Chem 1996; 271: 26706-26712.

$30 \mathrm{Ko} \mathrm{JH}$, Miyoshi E, Noda K, and et al. Regulation of the GnT-V promoter by transcription factor Ets-1 in various cancer cell lines. J Biol Chem 1999; 274: 22941-22948.

31 Oikawa M, Abe M, Kurosawa H, Hida W, Shirato K, Sato Y. Hypoxia induces transcription factor ETS-1 via the activity of hypoxia-inducible factor-1. Biochem Biophys Res Commun 2001; 289: 39-43.

32 Elbarghati L, Murdoch C, Lewis CE. Effects of hypoxia on transcription factor expression in human monocytes and macrophages. Immunobiology 2008; 213: 899-908.

33 Saito T, Miyoshi E, Sasai K, and et al. A secreted type of $\beta 1,6-N$ acetylglucosaminyltransferase V (GnT-V) induces tumor angiogenesis without mediation of glycosylation: a novel function of GnT-V distinct from the original glycosyltransferase activity. J Biol Chem 2002; 277: 17002-17008.

34 Nakahara S, Saito T, Kondo $\mathrm{N}$, and et al. A secreted type of $\beta 1,6 \mathrm{~N}$ acetylglucosaminyltransferase $\mathrm{V}(\mathrm{GnT}-\mathrm{V})$, a novel angiogenesis inducer, is regulated by $\gamma$-secretase. FASEB J 2006; 20: 2451-2459.

35 Wang $\mathrm{R}$, Zhang $\mathrm{YW}$, Zhang $\mathrm{X}$, and et al. Transcriptional regulation of APH-1A and increased $\gamma$-secretase cleavage of APP and Notch by HIF-1 and hypoxia. FASEB J 2006; 20: 1275-1277. 